\title{
PREVENCIÓN DEL ESTRÉS EN PROFESORES DEL DEPARTAMENTO DE MATEMÁTICAS Y ESTADÍSTICA - UFPS
}

Por:

Luz Marina Herrera León', Mawency Vergel Ortega', Henry Gallardo', Néstor Aristizabal

\section{RESUMEN}

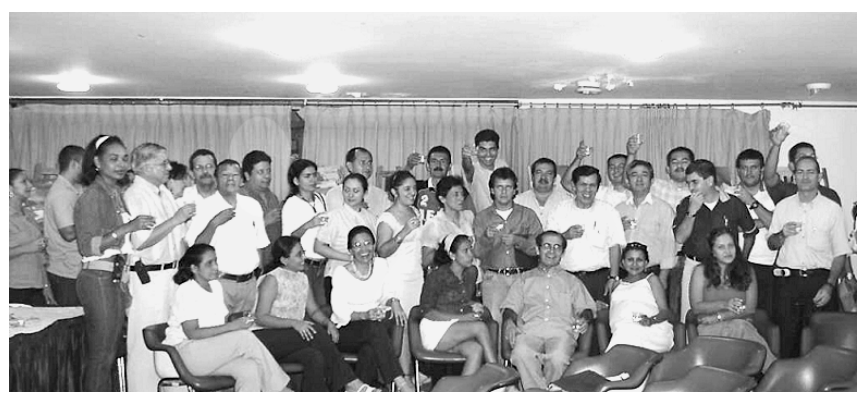

El Departamento de Matemáticas y Estadística de la UPFS realiza talleres de complementación académica en las áreas de estadística, cálculo y geometría. Sin embargo, teniendo en cuenta el número de cursos, el alto número de estudiantes, las nuevas políticas laborales y demás actividades académicasadministrativas de los docentes, se observaba un nivel de estrés en cada uno de ellos elevado, el cual tendía a convertirse en el mediano plazo, en un riesgo alto para la salud, la estabilidad familiar y emocional, toda vez que el estrés es considerado uno de los enemigos silenciosos con mayor influencia negativa en la salud del individuo. Por lo tanto, se hizo necesario la integración de los profesores, invitándolos a compartir actividades lúdicas, académicas y terapias psicológicas, que permitieron manejar el estrés y así mismo determinar, si el nivel de estrés de los profesores incide en rendimiento académico de los estudiantes. Se presenta el proyecto piloto con miras a proyectarse a toda la institución.

\section{ABSTRACT}

The Department of Mathematics and statistic had developed courses of academic complementation in the statistic areas, calculation and geometry. However, having in mind the number of courses, the high number of students, the new politicians labor and other academic-administrative activities of the educational ones, an stress level was observed in high each one of them, which spread to become the medium term, in a high risk for the health, the family and emotional stability, all time that the stress one of the silent enemies is considered with more influence negative in the individual's health, therefore, it became necessary the integration of the professors, to share activities amusing, academic and psychological therapies that allowed to manage the stress and likewise to determine if the level of the theachers' stress impacts in the students' academic yield. The project pilot is presented with an vision toward being projected to the whole institution.

Palabras claves: Estrés, Estrés docentes, tests de personalidad MMPI-2, Estudio de Percepción, Prueba del signo, Prueba de Mann- Whitney, Análisis de varianza.

\section{INTRODUCCIÓN}

El grupo de investigación en Estadística Aplicada GRAUNT- de la Universidad Francisco de Paula Santander, con una de sus líneas de investigación en estadística aplicada, participó en las convocatorias de FODESEP Y FINU, obteniendo la financiación del proyecto "El estrés en los profesores del Departamento de Matemáticas y Estadística". En este proyecto se buscó dar apoyo a los profesores para prevenir el estrés, enemigo oculto de la salud. Se midieron los niveles de estrés, utilizando los indicadores del NIOHS (Instituto Nacional de Salud y Seguridad Ocupacional de los Estados Unidos de América), al iniciar y terminar el proyecto, se caracterizaron a los profesores, se aplicó el test de personalidad MMPI-2, se observó la percepción que tenían los estudiantes de sus profesores y se determinó el análisis de varianza en los niveles de estrés, como también en el rendimiento académico de los estudiantes en los cursos que dirigían los profesores participantes en el proyecto.

1 Grupo de Investigación en Estadística Aplicada - biedomye@motilon.ufps.edu.co 
Prevención del estrés en profesores del departamento de Matemáticas y Estadistica - UFPS

\section{PROCEDIMIENTO Y METODOLOGÍA}

Al iniciar el programa, a los docentes del departamento participaron en cursos de inducción, donde se les presentó los planteamientos y objetivos del proyecto y mostramos las consecuencias fatales del estrés. También se enfatizó en que "no existe una formula sencilla e infalible que pueda curar el estrés". Se les explicó que se requieren acciones diversas para reducir las situaciones de sobrecarga.

Para ello era necesario "reaprender" a realizar las tareas cotidianas del hogar y/o el trabajo: programar las actividades para que no se acumulen ni se conviertan en factores estresantes, establecer prioridades claras, delegar responsabilidades, aprender a decir no a los compromisos que no se pueden cumplir, hacer bien y pronto lo que se puede hacer y olvidarse por completo de lo que no se puede, y asumir los grandes retos como secuencias de pequeños pasos. Se les informó que participarían en actividades lúdicas y las técnicas de relajación y actividades deportivas. Se les recomendó tener siempre en cuenta la famosa frase de Reinhold Niebuhr: "Señor, dadme el coraje para cambiar las cosas que debo, serenidad para aceptar las que no puedo cambiar, y sabiduría para reconocer la diferencia"[1].

Se midieron los niveles de estrés al iniciar y terminar el proyecto, utilizando los indicadores de NIOHS, determinando la varianza y observando el efecto de la capacitación en la que participaron los docentes. También se determinaron los indicadores de estrés mas comunes que presentaban los profesores para ayudarlos a combatir estas manifestaciones de estrés. Se caracterizaron los profesores para conocer su nivel económico, hobbies, estructura familiar y así poder analizar si existían situaciones que eran estresantes para unos y no lo eran para otros.

Se aplicó el test de personalidad MMPI-2[2] para observar rasgos específicos de personalidad, que de alguna manera podria afectar la información personal que se manejaba de cada uno de los docentes. Se analizó la percepción que tenían los estudiantes de sus profesores para evidenciar si estos se veían afectados por el estrés de sus profesores. Y finalmente se hizo el análisis de varianza del rendimiento académico de los estudiantes en los cursos que dirigían los profesores participantes en el proyecto, determinado la incidencia del estrés. Al finalizar el proyecto nuevamente se evaluaron los niveles de estrés.

\section{RESULTADOS}

Los resultados del trabajo realizado fueron los siguientes, se logró la participación y motivación de los profesores, se creó conciencia acerca del cuidado del bienestar físico y mental y el interés por estos temas fundamentales de bienestar universitario, se llegó a otros departamentos académicos de la universidad Departamentos de Física, Química, Biología, Fluidos y térmicas, Sistemas y Diseño Mecánico, donde se seleccionó un participante por cada departamento.

\section{Análisis de la encuesta de estrés}

Una vez recolectada la información, se procesó y analizó obteniendo los siguientes resultados: la encuesta aplicada sobre el estrés, nos muestra que el 6\% de los docentes del Departamento de Matemáticas y Estadística necesitaban tratamiento con especialista, un $29 \%$ de los docentes presentaban una situación de "alerta" de estrés y un 65\% de los docentes presentan niveles normales de estrés.

TABLA 1. Niveles de estrés de docentes del Dpto. de Matemáticas y Estadística - UFPS

\begin{tabular}{lcr}
\hline Niveles & Nú mero & $\%$ \\
\hline Niveles normales & 30 & 65 \\
Alerta de estrés & 14 & 29 \\
Necesidad de atención especializada & 6 & 6 \\
\hline Total & 50 & 100 \\
\hline
\end{tabular}

Las manifestaciones más comunes del estrés entre los docentes del Departamento Matemáticas y Estadística son: tensión muscular, quieren cambiar estilo de vida, 
Prevención del estrés en profesores del departamento de Matemáticas y Estadistica - UFPS

mal genio e irritación por pequeños inconvenientes, olvidan las cosas, se sienten cansados aunque haya dormido suficiente, se duermen pensando en los problemas o en lo que va hacer mañana, comen poco y toma mas tinto de lo normal, no se sienten eficientes con el trabajo, lloran o le cuentan a sus amigos sobre los problemas que le aquejan, se sienten insatisfechos con lo que hacen.

\section{Caracterización de los profesores}

Según la opinión de expertos en el tema, las características del trabajador asociadas a las condiciones de trabajo son la primera causa de estrés en el trabajo. Para evaluar esta opinión se aplicó unas encuestas que dió los siguientes resultados. Se observa en la muestra los participantes son aproximadamente igual, hombres (59\%) y (41\%) mujeres; La edad promedio de los profesores del Departamento de Matemáticas y Estadística es de 38 años. Los docentes participantes del proyecto son procedentes de Norte de Santander el 94\%, y el 6\% de otros Departamentos. Referente al estado civil:

TABLA 2. Persona con quien vive.

\begin{tabular}{lcc}
\hline Personas con quienes vive & Número & $\%$ \\
\hline Esposa e hijos & 35 & 70 \\
Padres & 9 & 18 \\
Pareja & 3 & 6 \\
Familiar & 2 & 4 \\
Solo & 1 & 2 \\
& & \\
\hline Total & 50 & 100 \\
\hline
\end{tabular}

Comentario: El 69\% de los profesores participantes del proyecto tienen hijos, en promedio tiene 2 hijos, el $31 \%$ no tiene; respecto a la vinculación son de Vinculación exclusiva: 45\% trabaja solo con la UFPS y el $55 \%$ trabajan además con otras instituciones.;

TABLA 3. Tipo de vinculación laboral con la UFPS

\begin{tabular}{lc}
\hline Vinculación laboral & $\%$ \\
\hline Docentes de planta & 6 \\
Contrato ocasional & 36 \\
Cátedra & 58 \\
\hline Total & 100
\end{tabular}

En la tabla 4 se observa que el 10\% de los docentes ganan mas de 2.000.000 de pesos, el 30\% gana entre 1.000 .000 y 2.000 .000 de pesos, el $28 \%$ gana entre 600.000 y 1.000 .000 , el $20 \%$ gana entre 400.000 y 600.000 y un $12 \%$ gana entre 200.000 y 400.000 .

TABLA 4. Ingresos mensuales

\begin{tabular}{ll}
\hline Ingresos (en miles de \$) & $\%$ \\
\hline Entre 200 y 400 & 12 \\
Entre 400 y 600 & 20 \\
Entre 600 y 1.000 & 28 \\
Entre 1.000 y 2.000 & 30 \\
Mas de 2.000 & 10 \\
\hline Total & 100 \\
\hline
\end{tabular}

Personas a cargo: El 84\% tiene personan a cargo y el $16 \%$ no tienen personas a cargo; Posesión de Bienes: El 24\% de los docentes tienen casa y carro, el $2 \%$ tienen casa y moto, el $34 \%$ solo tiene casa, el $14 \%$ tienen carro, el $6 \%$ tiene carro y el $20 \%$ tienen otros bienes; Medio de transporte en la tabla 5. para llegar a la Universidad se emplean los siguientes medios de transporte: $41 \%$ llega a la universidad en carro, el $39 \%$ en buseta, el $12 \%$ a pie, $6 \%$ en moto y un $2 \%$ en bicicleta;

TABLA 5. Medio de transporte

\begin{tabular}{lc}
\hline Tipo de trasporte & $\%$ \\
\hline Carro & 41 \\
Transporte público & 39 \\
A pie & 12 \\
Motocicleta & 6 \\
Bicicleta & 2 \\
\hline Total & 100 \\
\hline
\end{tabular}

El motivo para vincularse con la UFPS fue: el $36 \%$ por gusto a la enseñanza, $17 \%$ por desarrollo personal, 10\% por sentido de pertenencia a la UFPS, $10 \%$ por satisfacción personal, 10\% mejorar ingresos económicos, $1 \%$ por experiencia, $1 \%$ por status, $1 \%$ por oportunidad de empleo. 
Prevención del estrés en profesores del departamento de Matemáticas y Estadistica - UFPS

TABLA 6. Motivos para trabajar en la UFPS

\begin{tabular}{lc}
\hline Motivos & $\%$ \\
\hline Gusto por la enseñanza & 36 \\
Desarrollo personal & 17 \\
Sentido de pertenencia a la UFPS & 10 \\
Mejorar ingresos & 10 \\
Adquirir experiencia & 1 \\
Estatus profesional & 1 \\
Oportunidad dada & 1 \\
Otros & 24 \\
\hline Total & 100 \\
\hline
\end{tabular}

El trato con sus compañeros de trabajo es: excelente $20 \%$, buena $74 \%$ y $6 \%$ aceptable; Correo Electrónico: El $63 \%$ posee y el $37 \%$ no lo tiene; Las actividades que comparten juntos son: en la tabla 7 , el $26 \%$ comparten actividades recreativas y deportivas, el $18 \%$ estudian, $17 \%$ participan juntos en actividades académicas, el $11 \%$ dialogan de temas diferentes, $11 \%$ realizan trabajos de investigación, el $9 \%$ comparten actividades, $3 \%$ les gusta conversar, $3 \%$ comparten reuniones y $2 \%$ hacen cursos

TABLA 7. Actividades compartida de los docentes

\begin{tabular}{ll}
\hline Motivos & $\%$ \\
\hline Recreativa y deportivas & 26 \\
Estudio & 18 \\
Actividades académicas & 17 \\
Dialogo de temas diferentes & 11 \\
Trabajo de investigación & 11 \\
Compartir actividades & 9 \\
Conversar & 3 \\
Comparte reuniones & 3 \\
Curso & 2 \\
\hline Total & 100 \\
\hline
\end{tabular}

Consideran que la participación cuando integran equipos es: El 39\% es activa, 31 \% buena, $11 \%$ alegre, $11 \%$ de compromiso, $5 \%$ organizador y $3 \%$ que es esporádica. Las EPS mas utilizadas por los docentes son Saludcoop y el Seguro Social con un $29 \%$ cada una, 18\% con Coomeva, un $8 \%$ con la Fundación Medico Preventiva, un 2\% con Colsanitas y un $14 \%$ no proporciono la EPS utilizada.
TABLA 8. EPS utilizada para la atención en salud

\begin{tabular}{lc}
\hline Motivos & $\%$ \\
\hline Seguro Social & 29 \\
Saludcoop & 29 \\
Coomeva & 18 \\
Fundación medico preventiva & 8 \\
Colsanitas & 2 \\
No dio información & 14 \\
& \\
\hline Total & 100 \\
\hline
\end{tabular}

Se determinó que a todos les gusta leer y sus preferencias por el tipo de lectura son: el 30\% prefiere lecturas especializadas, el 18\% literaturas, el 15\% lecturas de psicología, el 10\% les gusta las lecturas científicas, el 9\% les gustan las lecturas variadas, el $7 \%$ leen el periódico, el 5\% lee novelas, $4 \%$ lecturas de metafísica, el $1 \%$ prefiere lecturas cristianas, el $1 \%$ prefiere lecturas de computación; Consumen alcohol: $53 \%$ consume alcohol y un $47 \%$ no y Consumo cigarrillo: $92 \%$ no fuman y un $8 \%$ si.

Análisis e interpretación del test de personalidad - MMPI-2.

Se hizo una interpretación global analizando los niveles de ansiedad, grado de introversión extroversión, tendencia idealista o realista, preocupaciones en torno al estado físico corporal y de salud, analizando cada escala para todo los sujetos, lo cual da unas tendencias dentro del colectivo de sujetos. Los profesores del Departamento de Matemáticas y Estadística de la UFPS. Se encontró que todos los profesores se encontraban dentro de los parámetros normales esperados. No se encontró ningún caso que amerite intervención psicológica en el inmediato o corto plazo. Cada uno de ellos fue entrevistado para darle a conocer su perfil de personalidad, resultado del Test aplicado.

Análisis de la encuesta sobre la percepción que tienen los estudiantes respecto a los profesores.

Los resultados obtenidos de la encuesta fueron los siguientes: Los estudiantes consideran que los docentes 
Prevención del estrés en profesores del departamento de Matemáticas y Estadistica - UFPS

más ansiedad a los estudiantes, los profesores disfrutan los chistes que hacen los estudiantes, los profesores difícilmente se concentran en el tema cuando los estudiantes hacen desorden. Consideran que los profesores son relativamente flexibles, Cuando un estudiante llega tarde o sale antes de terminar la clase el profesor le llama atención; los estudiantes consideran que a los profesores les gustaría que ellos entendieran rápidamente los temas explicados.

Cuando un estudiante no entiende, los profesores pacientemente vuelven a explicar; cuando los estudiantes llegan a clase, el profesor esta dispuesto a empezar la clase. En general, los profesores no lucen fatigados en clase, aceptan que los trabajos presentados por los estudiantes tengan algún error, toman con paciencia la lentitud de algunos estudiantes, no son malgeniados, las correcciones las hacen de buena forma. A los estudiantes les es fácil hacer consulta con los profesores.

Los profesores son comprensivos cuando los estudiantes no pueden cumplir con fechas de los trabajos y exámenes previos; exigen que, para iniciar la clase debe haber perfecto orden y silencio; no se quejan por el ruido que se produce alrededor; aceptan reclamos y excusas; Los profesores no se molestan por los chistes que hacen los estudiantes; los estudiantes consideran que pueden hacer preguntas sin temer que el profesor ridiculice las clases transcurren en calma y tranquilidad.

Análisis de varianza en el impacto de programa en el rendimiento académico de los estudiantes

Para esta parte del proyecto se utilizó la prueba de Mann-Whitney[3], es muy eficiente cuando las distribuciones que se comparan son continuas. Esta prueba mostró con un nivel de confiabilidad del 95\% que los niveles de estrés de los profesores no incidieron en el rendimiento académico de los estudiantes.
Análisis de varianza en Los niveles de estrés de los docentes

Al terminar el programa se evaluaron nuevamente las manifestaciones de estrés. Para evaluar el análisis de varianza en esta parte del proyecto, se utilizó la prueba del Signo, con esta prueba se mostró con un nivel de confiabilidad del 95\%, que los niveles de estrés de los profesores, si disminuyeron totalmente con la capacitación recibida.

\section{CONCLUSIONES}

Se aplico la prueba del signo para la varianza de los niveles de estrés la cual mostró con un nivel confiabilidad del $95 \%$, que los tratamiento a los que se sometieron los profesores en el proyecto, lograron disminuir de manera apreciable los niveles de estrés que presentaron el grupo en observación. Estos resultados evidencian que el ejercicio habitual, las actividades lúdicas y de relajación son técnicas que contrarrestan el efecto nocivo del estrés y son especialmente útiles, pues proporciona una forma de reducción para la agresividad y la tensión, mejorar el funcionamiento cardiovascular y genera un estado placentero de relajación después de cada práctica.

Es necesario someter a los profesores a programas de bienestar, para lograr disminuir los niveles de estrés y permitirles evaluar sus quehaceres, logrando de alguna manera replantearlos para tener un manejo acertado del estrés.

Se observó que los rasgos de los profesores no tenían relación con los niveles de estrés que manejaban. Estos resultados nos ayudó a entender de alguna manera, que ciertas condiciones que son estresantes para una persona, no lo son para otra.

En el estudio en cuestión los profesores presentaron un tipo personalidad normal que hacen más valido los resultados obtenidos. Presentaron niveles normales de ansiedad, grado de introversión, extroversión, 
Prevención del estrés en profesores del departamento de Matemáticas y Estadistica - UFPS

tendencia idealista o realista, preocupaciones en torno al estado físico corporal y de salud.

El proyecto logro integrar los profesores en un ambiente favorable para el trabajo en equipo facilitándose la labor académica e investigativa, y a tomar conciencia de la importancia del cuidado de la salud física y mental.

\section{AGRADECIMIENTOS}

El grupo de investigación GRAUNT, agradece a todos los profesores participantes en el proyecto, por su colaboración en la recolección de información, su puntualidad a todos los eventos organizados, por su apoyo, motivación, sugerencias y en particular por su esmero en continuar creando grupos de apoyo para prevenir el estrés. También agradecemos a FODESP y FINU, que con su financiación hizo posible la realización de este proyecto. A la O.N.G. INTERCIENCIAS y a INTERFISICAS en la realización de los talleres.

\section{REFERENCIA BIBLIOGRÁFICA}

[1]. ARIAS GALICIA, Fernando: Administración de Recursos humanos. $3^{a}$. Edición. México, Ed. Trillas, 1988.

[2]. RIVERA JIMENEZ, Ofelia: Interpretación clínica y psicodinamica del MMPI. México, Ed. Diana, 1989.

[3]. PAGANO, Robert R. Estadística para Ciencias del Comportamiento. Edición 5. México. Thomson Editores. 2002.

Fecha de recibido: Octubre 19 de 2005

Fecha de Aceptación: Noviembre 30 de 2005 